\title{
The cross sections of fusion-evaporation reactions: the most promising route to superheavy elements beyond $Z=118$
}

\author{
Khuyagbaatar Jadambaa ${ }^{1,2, \star}$ \\ ${ }^{1}$ Helmholtz Institute Mainz, 55099 Mainz, Germany \\ ${ }^{2}$ GSI Helmholtzzentrum für Schwerionenforschung, 64291 Darmstadt, Germany
}

\begin{abstract}
The synthesis of superheavy elements beyond oganesson $(\mathrm{Og})$, which has atomic number $Z=118$, is currently one of the main topics in nuclear physics. An absence of sufficient amounts of target material with atomic numbers heavier than californium $(Z=98)$ forces the use of projectiles heavier than ${ }^{48} \mathrm{Ca}(Z=20)$, which has been successfully used for the discoveries of elements with $Z=114-118$ in complete fusion reactions. Experimental cross sections of ${ }^{48} \mathrm{Ca}$ with actinide targets behave very differently to "cold" and "hot" fusion-evaporation reactions, where doubly-magic lead and deformed actinides are used as targets, respectively. The known cross sections of these reactions have been analysed compared to calculated fission barriers. It has been suggested that observed discrepancies between the cross sections of ${ }^{48} \mathrm{Ca}$-induced and other fusionevaporation reactions originate from the shell structure of the compound nucleus, which lies in the island of the stability. Besides scarcely known data on other reactions involving heavier projectiles, the most promising projectile for the synthesis of the elements beyond $\mathrm{Og}$ seems to be ${ }^{50} \mathrm{Ti}$. However, detailed studies of ${ }^{50} \mathrm{Ti},{ }^{54} \mathrm{Cr}$, ${ }^{58} \mathrm{Fe}$ and ${ }^{64} \mathrm{Ni}$-induced reactions are necessary to be performed in order to fully understand the complexities of superheavy element formation.
\end{abstract}

\section{Introduction}

The search for "hypothetical" nuclei from the island of stability, referred to as superheavy nuclei (SHN), which are predicted to be located at around proton number $Z=$ 114 and neutron number $N=184$ according to most theoretical calculations [1-3], is the one of the main goals of fundamental nuclear physics research. To date, isotopes of elements up to $Z=118$ and $N=177$ have already been discovered [4]. The heaviest elements $(Z=114-118)$ have been directly produced in ${ }^{48} \mathrm{Ca}$-induced reactions with actinide targets, first at Dubna Gas-Filled Recoil Separator (DGFRS) at the Flerov Laboratory of Nuclear Reactions in Dubna, Russia. The findings of these works were confirmed world-wide by different laboratories using the same reactions [5-15]. Recently, four elements with $Z=113,115,117$ and 118 have officially been named as nihonium $(\mathrm{Nh})$, moscovium $(\mathrm{Mc})$, tennessine (Ts) and oganesson $(\mathrm{Og})$, respectively [16].

Presently, the synthesis of elements beyond $\mathrm{Og}$ is the hot topic in the research field, despite that the predicted magic $Z=114$ has already been reached. However, an absence of sufficient amounts of target material with atomic numbers heavier than californium $(Z=98)$ currently prevents the use of ${ }^{48} \mathrm{Ca}$ for synthesis of elements beyond $\mathrm{Og}$. The predicted magic $N=184$ also remains out of reach due to limited access to both radioactive beams and appropriate target materials.

\footnotetext{
^e-mail: J.Khuyagbaatar@gsi.de
}

Of special interest is the element with $Z=120$, wherein some theories predict a stronger shell closure compared to the "classical" magic $Z=114$ i.e. a shift in the location of the center of the island of stability. Several attempts to synthesize the element with $Z=120$ have already been carried out using ${ }^{64} \mathrm{Ni}+{ }^{238} \mathrm{U}[17],{ }^{58} \mathrm{Fe}+{ }^{244} \mathrm{Pu}$ [18], ${ }^{54} \mathrm{Cr}+{ }^{248} \mathrm{Cm}$ [19], and ${ }^{50} \mathrm{Ti}+{ }^{249} \mathrm{Cf}$ [20] reactions. However, none of these studies reported observation of the element with $Z=120$, despite reaching sensitivity levels that would be sufficient to detect at least one event from any of the known ${ }^{48} \mathrm{Ca}$-induced reactions. This nonobservation can firstly be due to entrance-channel effects [21], as the coulomb repulsion forces of these reactions are significantly greater than those for ${ }^{48} \mathrm{Ca}$-induced reactions. Secondly, the survival probabilities of compound nuclei could also be smaller when compared with Og, according to most theoretical predictions [1-3]. Also, impacts of the double magicity $(Z=20$ and $N=28)$ of ${ }^{48} \mathrm{Ca}$ on capture, fusion and survival probabilities are still not yet fully understood [22] despite its effect on the reaction $Q$-value.

However, knowledge of the effect of these two processes remains poor, not only for elements beyond $\mathrm{Og}$ but also for lighter ones. For the observation of a single atom of a superheavy element, experiments must often be performed for several days, weeks or months [4-15, 1720, 23]. Therefore, choosing a suitable projectile-target combination and optimum beam energy for a reaction are of upmost importance to maximize the countable yield of heavy nuclei. 
The cross sections of ${ }^{48} \mathrm{Ca}$-induced fusionevaporations reactions with actinide targets remain at the level of pbarns as a function of $Z$ of the compound nucleus $\left(Z_{C N}\right)$, as opposed to those of "cold"- and "hot"fusion reactions (where doubly-magic lead and deformed actinides are used as targets, respectively), which decrease exponentially. It has already been suggested that such behaviour is associated with the survival probability of the compound nucleus [4].

In the present paper, a compilation of the known data on ER cross sections of the above mentioned reactions were performed and tested for a presence of any systematic trends. This would help to understand the discrepancy in the case of ${ }^{48} \mathrm{Ca}$, and may also be useful for the selection of preferable projectile and target combinations leading to elements beyond $\mathrm{Og}$.

\section{The cross sections of the fusion-evaporation reactions}

Since all of the SHN have been exclusively produced in fusion-evaporation reactions, their experimental cross sections may provide an important information on the preferable choice of projectile and target combinations for the synthesis of the as yet unknown SHN. The evaporation residue (ER) cross section of fusion reactions passing through a formation of compound nucleus $(\mathrm{CN})$ is often described by the three-term expression,

$$
\sigma_{E R}\left(E^{*}, J\right)=\sigma_{c a p}(E, J)\left[1-P_{Q F}(E, J)\right] W_{C N}\left(E^{*}, J\right),
$$

where $\sigma_{\text {cap }}$ is the cross section characterizing the formation of the di-nuclear system (capture) at collision energy $E, P_{Q F}$ is the fraction of di-nuclear systems not evolving via formation of $\mathrm{CN}$ with a compact shape i.e. splitting into two fragments (quasi-fission), and $W_{C N}$ is the survival probability of $\mathrm{CN}$ against fission through particle evaporation at the excitation energy of $E^{*}=E-Q$, where $Q$ is the energy necessary for the fusion. Terms $\sigma_{c a p}$ and $W_{C N}$ describe independent processes and have been studied substantially, both experimentally and theoretically.

A theoretical description of $P_{Q F}$, or its complementary $P_{C N}=1-P_{Q F}$, which characterizes the fusion probability, is not yet fully developed $[24,25]$. The main problem is the dynamical evolution of the multidimensional di-nuclear system leading to equilibration in all possible degrees of freedom, which is still not describable despite developments in modern theory and computing. Therefore, $P_{C N}$ is often extrapolated from the experimental observations of the nuclear reaction studies [26]. In the earlier studies, where mostly the light particles/ions were used as a projectile, the quasi-fission probability was shown to be negligible. With more massive ion beams with $Z>20$ that gave access to superheavy elements, a reduction of $P_{C N}$ has been observed due to quasi-fission. In reactions with strong coulomb repulsion forces between the collision partners $\left(Z_{p} Z_{t}>1600\right)$, the presence of quasi-fission was predicted [27]. As a result, $\sigma_{E R}$ for fusion reactions leading to the formation of SHN drastically decreases as function of $Z_{C N}$ or $Z_{p} Z_{t}$.

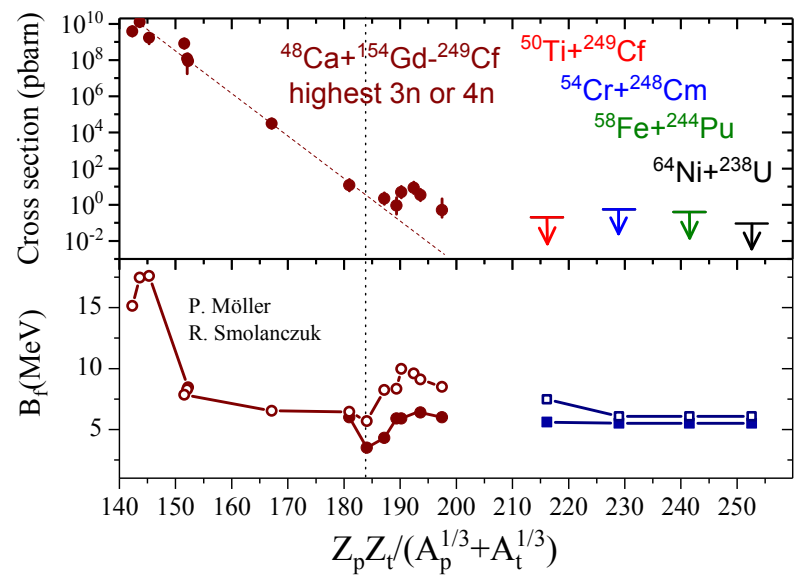

Figure 1. a) Compiled experimental ER cross sections of fusionevaporation reactions with ${ }^{208} \mathrm{~Pb}$ and ${ }^{238} \mathrm{U}$ targets, and ${ }^{48} \mathrm{Ca}$ projectiles [28-32]. Only maximum values of $\sigma_{1 n}$ and $\sigma_{5 n}$ are shown for ${ }^{208} \mathrm{~Pb}$ and ${ }^{238} \mathrm{U}$, respectively. Maximum values of either $\sigma_{3 n}$ or $\sigma_{4 n}$ from ${ }^{48} \mathrm{Ca}$-induced reactions with deformed Gd-Cf targets are shown. Arrows mark upper limits. Dashed lines are drawn to guide an exponential descent. b) Theoretical $B_{f}-S_{n}$ calculated within the macro-microscopic FRDM approach are given [3, 37].

\section{1 "Cold", "hot"and ${ }^{48}$ Ca-induced fusion reactions}

Compiled ER cross sections of three types of fusion reactions are shown in Fig. 1 as a function of $Z_{p} Z_{t} /\left(A_{p}^{1 / 3}+A_{t}^{1 / 3}\right)$ parameter which is equivalent to the Coulomb repulsion at contact between spherically shaped projectile and target nuclei. This or any other variables like effective entrance channel fissility are better for displaying the reaction entrance channel than $Z_{C N}$ or $Z_{p} Z_{t}$. As representatives of "cold", and "hot" fusion, the maxima cross sections of 1 and 5 neutron evaporation channels, $\sigma_{1 n}$ and $\sigma_{5 n}$, of reactions with ${ }^{208} \mathrm{~Pb}$ and ${ }^{238} \mathrm{U}$ targets, respectively, were compiled [28, 29, 32]. In the case of the ${ }^{48} \mathrm{Ca}$-induced reactions, only maximum of $\sigma_{3 n}$ or $\sigma_{4 n}$ with deformed targets were selected to test for any systematic trend. In this case the well-known orientation effect of the collision will be accounted for [24, 33, 34].

In the statistical model, the survival probability of the excited nucleus has an exponential dependence on the fission barrier, $B_{f}$, and neutron separation energy, $S_{n}$ and is often expressed as

$$
W_{C N}\left(E^{*}\right) \sim \prod_{i}^{x} e^{\left[\left(B_{f}-S_{n}\right) / T\right]_{i}},
$$

where $T$ and $x$ are the temperature and number of emitted neutrons from the $\mathrm{CN}$. The latter depends on available $\mathrm{E}^{*}$ of the nucleus populated at each stage.

The fission probability of the highly-excited heavy nucleus strongly increases as function of $E^{*}[35]$. Thus, the survival probability of the initial $\mathrm{CN}$ which has highest possible $\mathrm{E}^{*}$, against the fission (first-chance fission) is the most crucial process for residue formation $[25,36]$. Accordingly, $B_{f}-S_{n}$ value of the initial $\mathrm{CN}$ can be applied for comparative analysis of $W_{C N}$. The calculated $B_{f}-S_{n}$ 


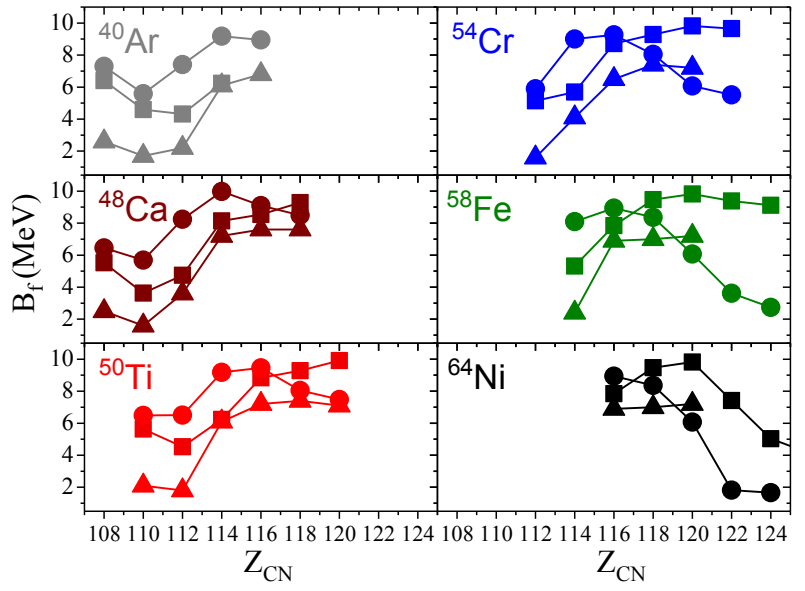

Figure 2. Calculated fission barriers of SHN, which can be formed as $\mathrm{CN}$ in ${ }^{40} \mathrm{Ar},{ }^{48} \mathrm{Ca},{ }^{50} \mathrm{Ti},{ }^{54} \mathrm{Cr},{ }^{58} \mathrm{Fe}$ and ${ }^{64} \mathrm{Ni}$-induced reactions with available actinide targets, ${ }^{226} \mathrm{Ra}^{249} \mathrm{Cf}$. The results from FRDM [37], $\mathrm{HBF}\left(\mathrm{Sk}^{*}\right)$ [40] and ETFSI [41] calculations are given by dots, squares and triangles, respectively.

of the initial $\mathrm{CN}$ corresponding to each reaction are shown in Fig. 1b) as a representative for the $W_{C N}$. Both variables have been extracted from the same mass FRDMcalculations $[3,37]$ to exclude systematic uncertainties due to the theoretical approaches.

Firstly, all three types of reactions have a decreasing trend of their $\sigma_{E R}$ as a function of the coulomb parameter i.e. $Z_{C N}$, except in the case of ${ }^{48} \mathrm{Ca}$ leading to elements heavier than $\mathrm{Hs}(Z=108)$. The decreasing slope is steeper in ${ }^{48} \mathrm{Ca}$-induced reactions compared to other two, which have a similar slopes. As mentioned above, the fusionevaporation reaction is the output of three consecutive physical processes. Accordingly, observed trends might have different origins. In "hot" fusion reactions where the coulomb repulsion force is smaller i.e., $Z_{p} Z_{t}<1600$, QF was believed to be negligibly small. However this criteria has been shown to be obsolete i.e. experimental evidence for the presence of $\mathrm{QF}$ in reactions with $Z_{p} Z_{t}<1600$ has been observed $[25,38]$. In "cold" fusion reactions where $Z_{p} Z_{t}>1600$, it is well known that QF dominates over fusion [27]. In addition, complied $\sigma_{1 n}$ of this type of reaction have been observed at energies below the fusion barrier, where $\sigma_{c a p}$ steeply decreases. Trends of $B_{f}-S_{n}$ for these two types of reactions do not show a significant difference (within the chosen scale of vertical axis) and vary smoothly as function of $Z_{C N}$. Therefore, the decreasing $\sigma_{E R}$ trend of "cold" fusion reactions could mostly be driven by entrance channel effects which can modify both capture cross sections and fusion probabilities.

In the case of ${ }^{48} \mathrm{Ca}, B_{f}-S_{n}$ values follow a U-shape as function of $Z_{C N}$, and initially decrease with increasing $Z_{C N}$ up to $\operatorname{Ds}(Z=110)$. This may explain the observed steeper decrease of $\sigma_{E R}$ compared to other two reactions. (i) After Ds, $B_{f}-S_{n}$ rises again as $B_{f}$ falls under the influence of the shell closure originating from the island of stability, thus impacting on $W_{C N}$ and increasing the $\sigma_{E R}$. Consequently,
$\sigma_{E R}$ of SHN beyond Ds that are enhanced compared to the expected exponential descent could be due to $W_{C N}$ [39].

To test the above-mentioned suggestion (i) initially proposed in Ref. [39], the results of other theoretical predictions of $B_{f}$ are examined. Fission barriers calculated within three different theoretical models are shown in Fig. 2. The results of FRDM compared to two other theoretical models, both microscopic self-consistent calculations performed within the Hartree-Fock-Bogoliubov (HFB) approximation with the $\mathrm{SkM}^{*}$ force [40] and Extended Thomas-Fermi plus Strutinsky Integral model (ETFSI) based on Skyrme SkSC4 functional [41], are shown.

Predicted fission barriers of $\mathrm{CN}$ formed in ${ }^{40} \mathrm{Ar},{ }^{50} \mathrm{Ti}$, ${ }^{54} \mathrm{Cr},{ }^{58} \mathrm{Fe}$ and ${ }^{64} \mathrm{Ni}$-induced reactions with ${ }^{226} \mathrm{Ra}^{-249} \mathrm{Cf}$ actinide targets are also shown in Fig. 2, in addition to those formed in ${ }^{48} \mathrm{Ca}$-induced reactions.

Overall, they all predict the existence of the island of stability despite their quantitative discrepancies. However, a precise prediction of an absolute $B_{f}$ is presently still not yet established for heaviest nuclei and results often differ from experimental ones by up to several $\mathrm{MeV}$ [42]. In spite of this, they all predict an increasing tendency of $B_{f}$ after passing Ds in the ${ }^{48} \mathrm{Ca}$ cases, thus supporting the arguments made in point (i) discussed above. One interesting feature can be noted in the case of $\mathrm{CN}$ formed in the ${ }^{40} \mathrm{Ar}+{ }^{248} \mathrm{Cm}$ reaction, where the calculations predict a similarly high $B_{f}$ as for the ${ }^{48} \mathrm{Ca}+{ }^{244} \mathrm{Pu}$ and $/$ or ${ }^{48} \mathrm{Ca}+{ }^{242} \mathrm{Pu}$ reactions where the $\sigma_{E R}$ are known. One has to emphasize that it will be interesting to measure $\sigma_{E R}$ of ${ }^{40} \mathrm{Ar}+{ }^{248} \mathrm{Cm}$ in order to help to understand the differences in the reaction mechanism.

Obvious differences in between theories can be found in cases where reactions lead to elements beyond Og because they predict differing values of $Z$ to be magic. For example, FRDM calculations predict a magic proton number at $Z=114$, compared to $Z=120$ in $\operatorname{HFB}\left(\mathrm{Sk}^{*}\right)$, as can be clearly seen in Fig. 2. If the latter prediction is correct, then one may expect to observe a more enhanced $W_{C N}$ of $\mathrm{CN}$ with $Z=120$ compared to $\mathrm{Cn}-\mathrm{Og}$, which may also increase the $\sigma_{E R}$. Thus, the synthesis of the element with $Z=120$ and measuring its production cross section will help to solve persistent discrepancies in the theoretical models.

\subsection{The reactions leading to formation of elements beyond 118}

For the synthesis of the element with $Z=120,{ }^{48} \mathrm{Ca}$ is can not presently be used, thus one needs to use a heavier projectiles like ${ }^{50} \mathrm{Ti},{ }^{54} \mathrm{Cr},{ }^{58} \mathrm{Fe},{ }^{64} \mathrm{Ni}$ etc.. The experimental cross-section sensitivities of four different reactions leading to formation of element $Z=120,{ }^{64} \mathrm{Ni}+{ }^{238} \mathrm{U}$ [17], ${ }^{58} \mathrm{Fe}+{ }^{244} \mathrm{Pu}$ [18], ${ }^{54} \mathrm{Cr}+{ }^{248} \mathrm{Cm}$ [19], and ${ }^{50} \mathrm{Ti}+{ }^{249} \mathrm{Cf}$ [20], are shown in Fig. 3 together with ${ }^{48} \mathrm{Ca}$ results. Values of calculated $B_{f}-S_{n}$ for CN calculated within the FRDM framework are also shown. To make a consistent comparison of $\sigma_{E R}$ to those discussed in the previous section, the known data (maximum of either $\sigma_{3 n}$ or $\sigma_{4 n}$ on ${ }^{50} \mathrm{Ti},{ }^{54} \mathrm{Cr}$, 
${ }^{58} \mathrm{Fe}$ and ${ }^{64} \mathrm{Ni}$-induced reactions with deformed Gd-Cf targets are compiled.

As seen from Fig. 3 data on these reactions are scarce. A decreasing trend can only be drawn in the case of ${ }^{50} \mathrm{Ti}$, which falls off much steeper than for ${ }^{48} \mathrm{Ca}$ (cf. Fig. 3a). However, it is probably not a realistic case for extracting a slope as the ER from the ${ }^{50} \mathrm{Ti}+{ }^{176} \mathrm{Yb}$ is an extremely neutron-deficient isotope of $U(Z=92)$ [43], and thus $W_{C N}$ may be strongly reduced.

This is also the case for the ${ }^{48} \mathrm{Ca}+{ }^{181} \mathrm{Ta}$ reaction (see Fig. 3a), where recently measured upper limit [44] is not following the extrapolated exponential. Therefore, one can assume a similar slope for all reactions including ${ }^{48} \mathrm{Ca}$ data, although these need to be measured. Extrapolated exponentials, fitted to the known data of the particular reaction and assuming the same slope as for ${ }^{48} \mathrm{Ca}$, are shown in Fig 3a.

In all four reactions leading to $Z=120$ formation discussed here $\left({ }^{64} \mathrm{Ni}+{ }^{238} \mathrm{U},{ }^{58} \mathrm{Fe}+{ }^{244} \mathrm{Pu},{ }^{54} \mathrm{Cr}+{ }^{248} \mathrm{Cm}\right.$, and ${ }^{50} \mathrm{Ti}+{ }^{249} \mathrm{Cf}$ ), one might expect a similar increase of $W_{C N}$ due to shell effects originating from the island of stability as seen in ${ }^{48} \mathrm{Ca}$-induced reactions (discussed in (i)). The $\mathrm{CN}$ is the same in all cases except for ${ }^{50} \mathrm{Ti}+{ }^{249} \mathrm{Cf}$, where the $\mathrm{CN}$ has three fewer neutrons, but the $B_{f}$ values are similar. From the known ${ }^{48} \mathrm{Ca}$ cross sections, leading to the formation of $\mathrm{Fl}-\mathrm{Og}$, this gain in cross section via the $W_{C N}$ enhancement can be estimated to be roughly two orders of magnitude higher than an exponential descent. One might then tentatively expect a similar gain in $\sigma_{E R}$ with respect to an exponential extrapolated, to be observed in reactions leading to $Z=120$.

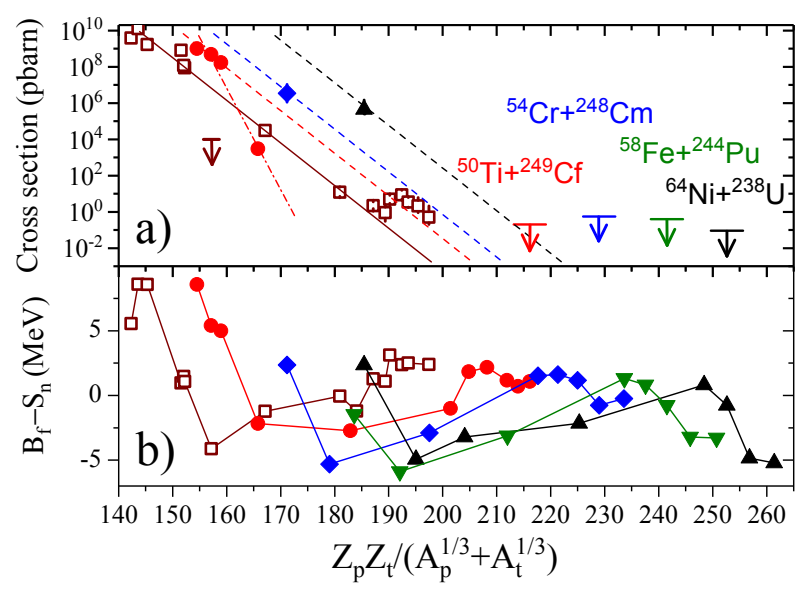

Figure 3. a) The compiled maximum of either $\sigma_{3 n}$ or $\sigma_{4 n}$ $[28,29,32,43,45]$ and b) calculated CN's $B_{f}-S_{n}$ within the FRDM model $[3,37]$ are given for ${ }^{48} \mathrm{Ca}$ (open symbols), ${ }^{50} \mathrm{Ti}$ (dots), ${ }^{54} \mathrm{Cr}$ (rhombuses), ${ }^{58} \mathrm{Fe}$ (down-triangles) and ${ }^{64} \mathrm{Ni}$ (uptriangles) induced reactions with deformed Gd-Cf targets. a) Cross-section sensitivities are marked by arrows [17-20, 44]. Solid line is drawn to guide an exponential descent of $\sigma_{E R}$ in ${ }^{48} \mathrm{Ca}$-induced reactions up to formation of $\mathrm{Hs}$ and continued to higher $Z C N$. Dashed-dotted line shows a trend of known $\sigma_{E R}$ for ${ }^{50} \mathrm{Ti}$-induced reactions. Dashes lines are the same as the solid one but shifted to fit known $\sigma_{E R}$ of the particular reaction. See text for details
As the coulomb forces increase significantly when changing from ${ }^{48} \mathrm{Ca}$ to a heavier projectile, the extrapolated $\sigma_{E R}$ decrease dramatically for a fixed $Z_{C N}$. Accordingly, reactions involving projectile and target combinations with the smallest coulomb forces are preferable for synthesizing element $Z=120$. Although the resulting $\sigma_{E R}$ estimate for the ${ }^{50} \mathrm{Ti}+{ }^{249} \mathrm{Cf}$ reaction of up to several fbarns $\left(10^{-3}\right.$ pbarns $)$ is not very encouraging and is about two-three orders of magnitude smaller than those for ${ }^{48} \mathrm{Ca}$ induced reactions, the true cross section could be quite different and therefore it is of great importance that the values be measured experimentally.

Note that the use of ${ }^{50} \mathrm{Ti}$ as a projectile also gives access to SHN with $Z=114-118$, whereas the range of accessible SHN with heavier projectiles appears to be even more limited, within the current capabilities and level of understanding of the fusion-evaporation reaction.

\section{Summary and outlook}

The compiled ER cross sections of three different types of fusion-evaporation reaction were comparatively analysed relative to the calculated fission barriers ( survival probability) from three different theoretical models. All three types of reaction (namely "cold", "hot" and ${ }^{48} \mathrm{Ca}-$ induced) show a similar decreasing trend of the ER cross sections as functions of the proton numbers in the compound nucleus. The known discrepancy in ER cross sections of ${ }^{48} \mathrm{Ca}$ with actinide targets were suggested to occur due to an enhanced survival probability of compound nuclei originating from shell closures associated with the island of stability, shown independently by the different theoretical frameworks discussed here.

A similarly enhanced survival probability for reactions leading to $Z=120$ may also be expected, providing an important testing ground for theories which, although they interpret the behaviour of ${ }^{48} \mathrm{Ca}+$ actinide results well, may not produce an accurate description for heavier systems. Experimental data on ER cross sections of reactions involving heavier projectiles and deformed targets, which are necessary for reaching $Z=120$, are very scarce. Thus, more measurements must be made.

Based on the present analysis, the main differences in the ER cross section of reactions ${ }^{64} \mathrm{Ni}+{ }^{238} \mathrm{U},{ }^{58} \mathrm{Fe}+{ }^{244} \mathrm{Pu}$, ${ }^{54} \mathrm{Cr}+{ }^{248} \mathrm{Cm}$, and ${ }^{50} \mathrm{Ti}+{ }^{249} \mathrm{Cf}$ leading to element with $Z=$ 120 could be strongly due to their entrance channel effects. One can argue that the latter reaction with smallest $Z_{p} Z_{t}$ might be the most preferable for a making the superheavy element with $Z=120$. For a final conclusion to be made on which reactions are preferable, further investigations of fusion and quasi-fission processes must be performed.

\section{References}

[1] A. Sobiczewski et al., Phys. Lett. 22, 500 (1966).

[2] W.D. Myers and W.J. Swiatecki, Nucl. Phys. 81, 1 (1966).

[3] P. Möller et al., At. Data. Nucl. Data. Tab. 59, 185 (1995). 
[4] Yu.Ts. Oganessian and V.K. Utyonkov, Nucl. Phys. A 944, 62 (2015)

[5] S. Hofmann et al., Eur. Phys. J. A 32, 251 (2007).

[6] L. Stavsetra et al., Phys. Rev. Lett. 103, 132502 (2009).

[7] R. Eichler et al., Radiochim. Acta 98, 133 (2010).

[8] Ch.E. Düllmann et al., Phys. Rev. Lett. 104, 252701 (2010).

[9] P.A. Ellison et al., Phys. Rev. Lett. 105, 182701 (2010).

[10] J.M. Gates et al., Phys. Rev. C 83, 054618 (2011).

[11] S. Hofmann et al., Eur. Phys. J. A 48, 62 (2012).

[12] A. Yakushev et al., Inorg. Chem. 53, 1624 (2014).

[13] D. Rudolph et al., Phys. Rev. Lett. 111, 112502 (2013).

[14] J. Khuyagbaatar et al., Phys. Rev. Lett. 112, 172501 (2014).

[15] D. Kaji et al,. J. Phys. Soc. Jpn. 86, 034201 (2017).

[16] L. Öhrström and J. Reedijk, Pure and Applied Chemistry $\mathbf{8 8}, 1225$ (2016).

[17] S. Hofmann et al., GSI Scientific Report-2007, 131 (2008).

[18] Yu.Ts. Oganessian et al., Phys. Rev. C 79, 024603 (2009).

[19] S. Hofmann et al., GSI Scientific Report-2011, 205 (2012).

[20] Ch.E. Düllmann et al., to be published.

[21] M.G. Itkis and A. Ya. Rusanov, Phys. Part. Nuclei 29, 160 (1998).

[22] C. Simenel et al., Phys. Lett. B 710, 607 (2012).

[23] J. Khuyagbaatar et al., GSI Scientific Report-2012, 131 (2013).
[24] K. Nishio et al.,Phys. Rev. C 82, 024611 (2010).

[25] J. Khuyagbaatar et al., Phys. Rev. C 91, 054608 (2015).

[26] M.G. Itkis et al., Nucl. Phys. A 787, 150 (2007).

[27] W.J. Swiatecki, Phys. Scr. 24, 113 (1981).

[28] http://nrv.jinr.ru/nrv/

[29] http://www.nndc.bnl.gov/

[30] K.E. Gregorich et al., Lawrence Berkeley National Laboratory, Berkeley, CA, USA, LBNL-Report 63617 (2007)

[31] S. Hofmann, Russ. Chem. Rev. 78, 1123 (2009).

[32] J. Khuyagbaatar, unpublished data for $3 \mathrm{n}$ cross section of the ${ }^{48} \mathrm{Ca}+{ }^{197} \mathrm{Au}$.

[33] D.J. Hinde et al., Phys. Rev. Lett. 74, 1295 (1995).

[34] K. Nishio et al.,Phys. Rev. C 77, 064607 (2008).

[35] R. Vandenbosch and J. R. Huizenga, Nuclear Fission, Academic, New York, (1973).

[36] R. Yanez et al., Phys. Rev. Lett. 112, 152702 (2014).

[37] P. Möller et al., Phys. Rev. C 79, 064304 (2009).

[38] A.C. Berriman et al., Nature (London) 413, 144 (2001).

[39] Yu.Ts. Oganessian, J. Phys. G 34, R165 (2007).

[40] A. Staszczak et al., Phys. Rev. C, 87, 024320 (2013).

[41] A. Mamdouh et al., Nucl. Phys. A 679, 337 (2001).

[42] A. Baran et al., Nucl. Phys. A, 944442 (2015).

[43] J. Khuyagbaatar et al., Phys. Rev. Lett. 115, 242502 (2015).

[44] A. Mistry, private communication.

[45] D.A. Mayorov et al., Phys. Rev. C 92, 054601 (2015). 